\title{
Electronic properties of mechanically induced kinks in single-walled carbon nanotubes
}

\section{Citation}

Bozovic, Dolores, M. Bockrath, Jason H. Hafner, Charles M. Lieber, Hongkun Park, and M. Tinkham. 2001. "Electronic Properties of Mechanically Induced Kinks in Single-Walled Carbon Nanotubes." Applied Physics Letters78 (23): 3693-95. https://doi.org/10.1063/1.1377316.

\section{Permanent link}

http://nrs.harvard.edu/urn-3:HUL.InstRepos:41534235

\section{Terms of Use}

This article was downloaded from Harvard University's DASH repository, and is made available under the terms and conditions applicable to Other Posted Material, as set forth at http:// nrs.harvard.edu/urn-3:HUL.InstRepos:dash.current.terms-of-use\#LAA

\section{Share Your Story}

The Harvard community has made this article openly available.

Please share how this access benefits you. Submit a story.

Accessibility 


\title{
Electronic properties of mechanically induced kinks in single-walled carbon nanotubes
}

\author{
Dolores Bozovic ${ }^{\mathrm{a})}$ and M. Bockrath \\ Department of Physics, Harvard University, Cambridge, Massachusetts 02138 \\ Jason H. Hafner, Charles M. Lieber, and Hongkun Park \\ Department of Chemistry and Chemical Biology, Harvard University, Cambridge, Massachusetts 02138 \\ M. Tinkham \\ Department of Physics, Harvard University, Cambridge, Massachusetts 02138
}

(Received 27 December 2000; accepted for publication 9 April 2001)

\begin{abstract}
We have used an atomic-force microscope tip to mechanically buckle single-walled carbon nanotubes. The resistance of the induced defects ranged from 10 to $100 \mathrm{k} \Omega$ and varied with the local Fermi level, as determined by scanned-gate microscopy. By forming two closely spaced defects on metallic nanotubes, we defined quantum dots less than $100 \mathrm{~nm}$ in length. These devices exhibited single-electron charging behavior at temperatures up to $\sim 165 \mathrm{~K}$. () 2001 American Institute of Physics. [DOI: 10.1063/1.1377316]
\end{abstract}

Depending on their radius and chirality, single-walled carbon nanotubes can be either one-dimensional (1D) metals or semiconductors. Initial experiments performed on metallic nanotubes concentrated on tubes that exhibited ballistic behavior. ${ }^{1-4}$ Recently, however, the role of native defects ${ }^{5}$ and artificially induced bending ${ }^{6}$ in transport properties of nanotubes has received considerable attention. Theoretical studies of the effects of mechanical deformation on electrical properties of carbon nanotubes have shown that localized distortions of the lattice should cause an increase in electron backscattering, increasing the resistance of the tubes. ${ }^{7-11}$

In this letter, we present experimental data that demonstrate this predicted increase of electron backscattering due to mechanically induced lattice deformations. We have used an atomic-force microscope (AFM) tip to bend nanotubes and create kinks at controlled sites along the tubes. ${ }^{12,13}$ Electron backscattering by the induced defects was then studied using scanned-gate microscopy (SGM). Further, we made nanoscale devices by creating kinks separated by $\sim 50 \mathrm{~nm}$, forming intratube quantum dots that exhibited Coulomb blockade behavior at temperatures up to $\sim 165 \mathrm{~K}$.

The substrate used in this work was degenerately doped silicon with a $1 \mu \mathrm{m}$ oxide layer. Nanotubes made by the laser arc-discharge method ${ }^{14}$ were ultrasonically suspended in ethylene dichloride and deposited on the substrate, while nanotubes made by chemical-vapor deposition (CVD) were grown directly on the surface of the chips. ${ }^{15,16}$ The nanotubes were inspected using a tapping-mode AFM, and tubes $\sim 1$ $\mathrm{nm}$ in height were located relative to preexisting alignment marks. Gold electrodes were then patterned over the nanotubes using standard e-beam lithography.

After attaching leads to the nanotubes, we used an AFM tip to manipulate the tubes on the surface of the substrate,,${ }^{17,18}$ while simultaneously performing transport measurements. To manipulate the nanotubes, we brought the AFM tip to the side of the tube and set its oscillation ampli-

${ }^{a)}$ Electronic mail: dbozovic@rsj.harvard.edu tude to zero. We then disengaged the feedback loop and further extended the piezoelectric scanner by $\sim 100 \mathrm{~nm}$, loading the tip with a force of $\sim 1-3 \mu \mathrm{N}$. Nanotubes were then manipulated by dragging the AFM tip across them. Creating a kink in a nanotube required pushing it a few hundred $\mathrm{nm}$ in the direction perpendicular to its axis. Figures 1 and 2 show images of samples taken after AFM manipulations have been performed. In Figs. 1(b) and 1(d), we show height scans of buckled tubes, taken along the lines indicated in Figs. 1(a) and 1(c). Kinks in the nanotubes show sharp increases in height compared to the unperturbed segments of the tubes, indicating that localized distortions have been induced.

Upon sharp bending with an AFM tip, nanotube resistances were found to increase by $10-100 \mathrm{k} \Omega$ and showed a
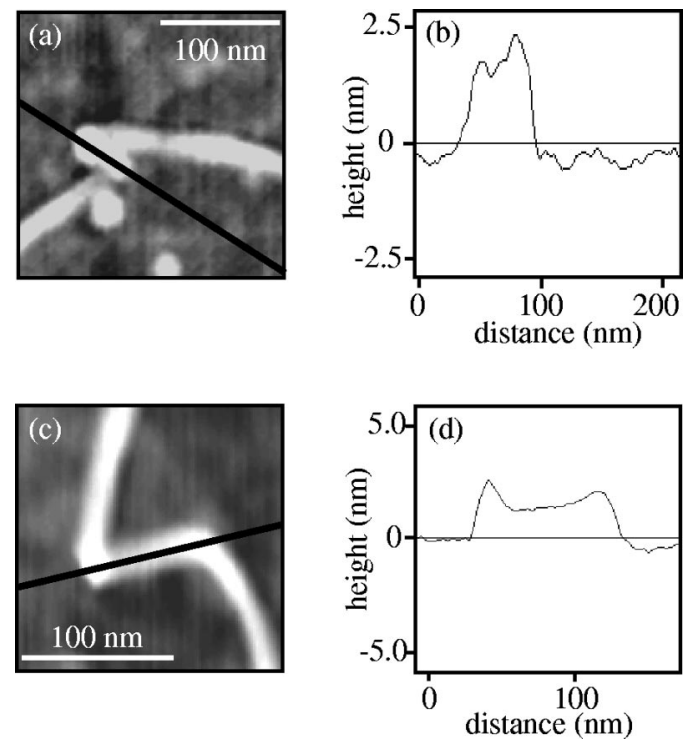

FIG. 1. Two kinks were mechanically induced in each of these samples. (a) and (c) show AFM images of samples 1 and 2, respectively, taken after such manipulations; (b) and (d) show the corresponding height scans, taken along the indicated lines. Sharp height increases can be observed at spots where the tubes have buckled. In (b), the two kinks are difficult to resolve separately, since they are closely spaced. 

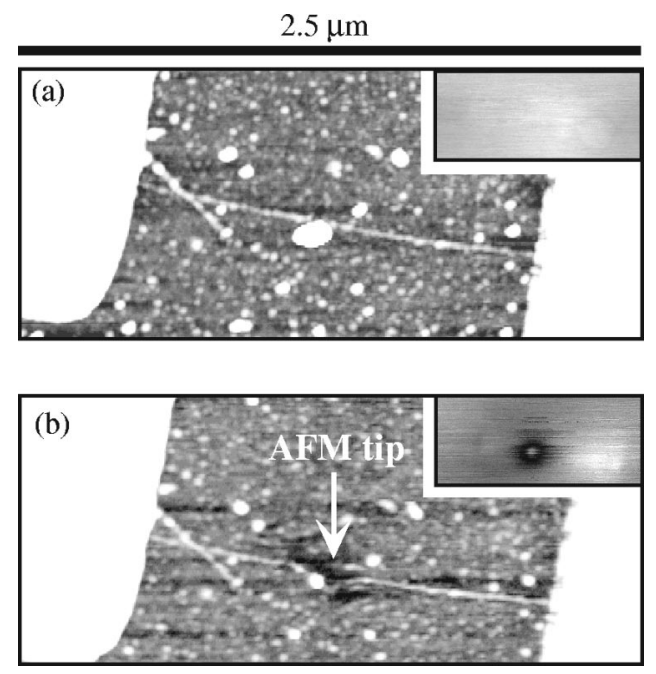

FIG. 2. Effect of AFM manipulation on transport properties of a carbon nanotube. (a) a tapping-mode AFM image of sample 3 before manipulation; (b) a scanned-gate image of the same sample; (c) the nanotube has been bent by AFM manipulation; (d) a dark ring-shaped modulation has appeared on the scanned-gate image at the deformed area.

strong dependence on the applied back-gate voltage. To further investigate this behavior, we used SGM, a local probe capable of detecting single scattering centers in nanotubes. ${ }^{19,20}$ In this approach, an AFM tip is used as a local gate that is scanned over the sample. Applying different voltages to the tip locally modulates the electrostatic potential on the nanotube, leading to a local shift in the Fermi level $E_{F}$. Plotting the conductance versus tip position generates a spatially resolved image of the effect of this perturbation. By performing SGM both before and after AFM manipulations, we directly observed changes in the electronic properties caused by kinks or sharp bends in the nanotubes.

Results of one such measurement are shown in Fig. 2. Figure 2(a) shows an unperturbed metallic nanotube contacted by gold leads, and the inset shows the corresponding scanned gate image. We then pushed the nanotube with an AFM tip in the direction perpendicular to the tube axis. Figure 2(b) shows the resulting bend in the nanotube, and its inset shows an SGM image taken immediately after this manipulation. A dark ring-like modulation [scanned-gate (SG) ring] has appeared in the area where the tube has been deformed. In the work of Bockrath et al.,${ }^{5}$ these SG rings were interpreted as signatures of resonant scattering by intratube defects in the as-grown nanotubes. Although SGM cannot resolve details of the lattice distortion on an atomic scale, the data do indicate that the scattering area is localized on a scale less than or equal to $\sim 100 \mathrm{~nm}$, which is the resolution limit imposed by the radius of curvature of the AFM tip. Furthermore, SGM images taken on a number of similarly prepared samples indicate that AFM manipulation leading only to a gradual bending of the nanotubes does not lead to SG rings. This is consistent with theoretical calculations, ${ }^{11,18}$ which indicate that mechanical distortions of tubes do not significantly affect the conductance unless the strain is large enough to change the local bond order or arrangement.

Next, we show the results of placing two kinks on a single metallic nanotube, thereby engineering small quantum dots at controlled positions along the tube. Although the radius of curvature of the AFM tip limits the spacing between Downloaded 19 May 2006 to 131.215.225.175. Redistribution subje
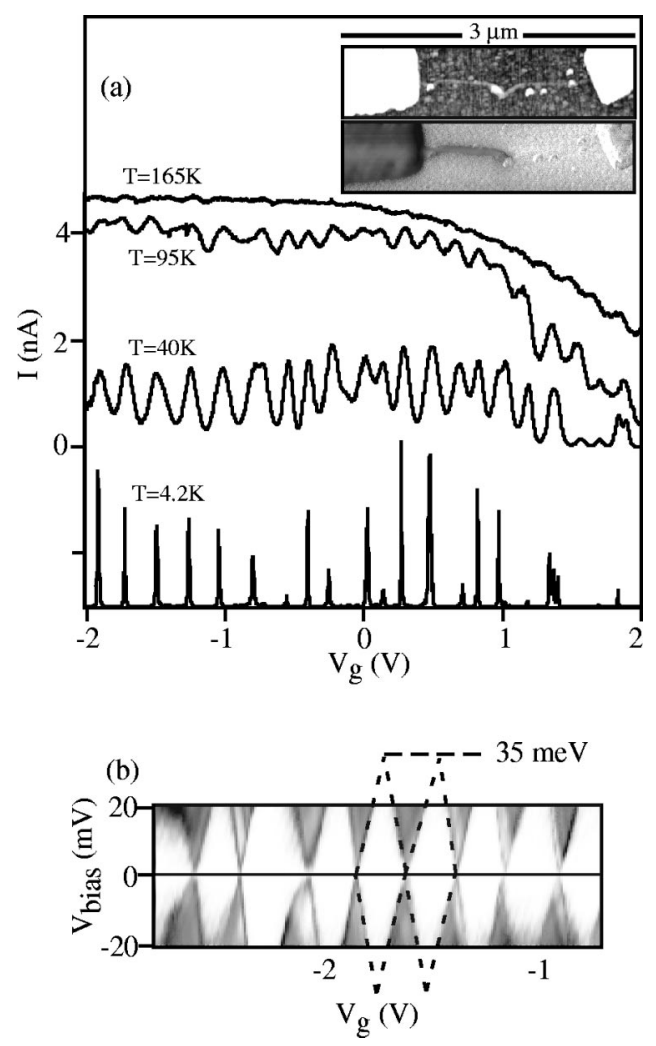

FIG. 3. (a) Current vs gate voltage sweeps, taken at $V_{\text {bias }}=1 \mathrm{mV}$. These data were taken on sample 1 [pictured in Fig. 1(a)], at temperatures of 4.2, 40, 95 , and $165 \mathrm{~K}$. The curve taken at $4.2 \mathrm{~K}$ has been offset for clarity. In the inset, we show a zoomed-out image of sample 1 on the top, and the corresponding EFM scan on the bottom. (b) A gray-scale plot of conductance vs bias and gate voltages, measured at $4.2 \mathrm{~K}$. White areas in the graph indicate the Coulomb blockade regime.

defects, we have been able to reproducibly make samples with two defects less than $100 \mathrm{~nm}$ apart. In Fig. 1, we show images of two such devices, with spacings of $\sim 50$ and 100 $\mathrm{nm}$. The nanotubes used here were grown by the CVD technique, and AFM manipulations were performed prior to depositing the electrodes on top. Without being anchored by the leads, the ends of a nanotube can slide along the surface of the chip during AFM manipulation. In this way, one could still obtain the localized distortions due to the two kinks, while minimizing the strain along the rest of the nanotube.

In Fig. 3(a), we show a plot of the low-bias conductance as a function of the back-gate voltage for sample 1, shown in Fig. 1(a). Data displayed in Fig. 3(a) were taken at various temperatures and found to exhibit periodic Coulomb charging peaks. As can be seen from this graph, periodic charging behavior can be detected at temperatures up to $165 \mathrm{~K}$. Furthermore, we can observe from the plots in Fig. 3(a) that the nanotube conductance is modulated by gate voltage, even though the nanotube is metallic. The induced kinks are, therefore, acting as gate-tunable scattering centers, allowing us to controllably tune the conductance of a metallic nanotube device.

In Fig. 3(b), we show a gray-scale plot of $d I / d V$ measured as a function of both the bias and gate voltages. The data show a repeated diamond pattern characteristic of single-electron charging. Extrapolation from the graph (as shown by the dashed lines for two typical diamonds) indicates that the Coulomb gap is on the order of $30-50 \mathrm{meV}$. to AIP license or copyright, see http://apl.aip.org/apl/copyright.jsp 
This maximal Coulomb gap corresponds to $(U+\Delta E)$, where $U$ is the charging energy and $\Delta E$ is the energy-level spacing. Following previous literature on the subject, ${ }^{1,2,21}$ we use $\Delta E \sim 0.5 \mathrm{eV} / L(\mathrm{~nm})$ and $U \sim 1.4 \mathrm{eV} / L(\mathrm{~nm})$ to estimate that these energies correspond to a 1D quantum dot of length $L$ $\sim 40-70 \mathrm{~nm}$. This matches the length of the short segment defined by the two kinks [see Fig. 1(a)], which is consistent with our assumption that the induced defects are acting as tunneling barriers, and thus defining a quantum dot.

In order to verify that it is indeed the two artificially created defects that are defining the charging behavior, we performed an electrostatic AFM (EFM) measurement ${ }^{19}$ on the nanotube. A conducting AFM tip, held at a constant voltage, is driven near its resonant frequency and scanned over the surface of the sample. The local electrostatic potential of the sample affects the force exerted on the conducting tip and shifts the resonant frequency of the cantilever. Detecting these frequency shifts thus provides a map of the electrostatic potential of the surface. After applying a bias voltage to a nanotube, one can use EFM imaging to detect voltage drops along the length of the tube. In the inset of Fig. 3(a), we show a larger area AFM image of sample 1 and the corresponding EFM scan. A sharp voltage drop occurs precisely at the spot where the nanotube has buckled (the two kinks cannot be resolved separately, since they are spaced by only $\sim 50 \mathrm{~nm}$ ). This indicates that the induced pair of kinks is dominating electron transport in the nanotubes and causing the observed charging behavior.

The experimental results reported here demonstrate that defects in nanotubes can be artificially induced using AFM manipulation, and that they can then be used to fabricate nanoscale devices on carbon nanotubes. In particular, we were able to make samples with two kinks that were less than $100 \mathrm{~nm}$ apart. These devices showed quantum-dot behavior up to temperatures of well over $100 \mathrm{~K}$. Fabricating quantum-dot devices with tunable conductances and operable at easily achievable temperatures opens up possibilities in future molecular electronics applications.
This work was supported in part by NSF Grant Nos. DMR-00-72618, DMR-98-0936, PHY-98-71810, and ONR Grant No. N00014-96-0108, the Dreyfus Foundation, and the Research Corporation (H.P.).

${ }^{1}$ S. J. Tans, M. H. Devoret, H. Dai, A. Thess, R. E. Smalley, L. J. Geerligs, and C. Dekker, Nature (London) 386, 474 (1997).

${ }^{2}$ M. Bockrath, D. H. Cobden, P. L. McEuen, N. G. Chopra, A. Zettl, A. Thess, and R. E. Smalley, Science 275, 1922 (1997).

${ }^{3}$ C. Dekker, Phys. Today 52, 22 (1999).

${ }^{4}$ T. W. Odom, H. Jin-Lin, P. Kim, and C. M. Lieber, Nature (London) 391, 62 (1998).

${ }^{5}$ M. Bockrath, W. Liang, D. Bozovic, J. H. Hafner, C. M. Lieber, M. Tinkham, and H. Park, Science 291, 283 (2000).

${ }^{6}$ T. W. Tombler, C. W. Zhou, L. Alexseyev, J. Kong, H. J. Dai, L. Lei, C. S. Jayanthi, M. J. Tang, and S. Y. Wu, Nature (London) 405, 769 (2000).

${ }^{7}$ B. I. Yakobson, C. J. Brabec, and J. Bernholc, Phys. Rev. Lett. 76, 2511 (1996).

${ }^{8}$ B. V. Pan, W. S. Yang, and J. Yang, Phys. Rev. B 62, 12652 (2000).

${ }^{9}$ P. Yang and J. Han, Phys. Rev. Lett. 85, 154 (2000).

${ }^{10}$ P. Zhang, P. E. Lammert, and V. H. Crespi, Phys. Rev. Lett. 81, 5346 (1998).

${ }^{11}$ A. Rochefort, P. Avouris, F. Lesage, and D. R. Salahub, Phys. Rev. B 60, 13824 (1999).

${ }^{12}$ H. W. C. Postma, M. de Jong, Z. Yao, and C. Dekker, Phys. Rev. B 62, 10653 (2000).

${ }^{13}$ M. R. Falvo, G. J. Clary, R. M. I. Taylor, V. Chi, F. P. J. Brooks, S. Washburn, and R. Superfine, Nature (London) 389, 582 (1997).

${ }^{14}$ These nanotubes were synthesized in the laboratory of Professor R. E. Smalley.

${ }^{15}$ J. H. Hafner, M. J. Bronikowski, B. R. Azamian, P. Nikolaev, A. G. Rinzler, D. T. Colbert, K. A. Smith, and R. E. Smalley, Chem. Phys. Lett. 296, 195 (1998).

${ }^{16}$ J. H. Hafner, C. L. Cheung, and C. M. Lieber, J. Am. Chem. Soc. 121, 9750 (1999).

${ }^{17}$ T. Hertel, R. Martel, and P. Avouris, J. Phys. Chem. B 102, 910 (1998).

${ }^{18}$ P. Avouris, T. Hertel, R. Martel, T. Schmidt, H. R. Shea, and R. E. Walkup, Appl. Surf. Sci. 141, 201 (1999).

${ }^{19}$ A. Bachtold, M. Fuhrer, S. Plyasunov, M. Forero, E. H. Anderson, A. Zettl, and P. L. McEuen, Phys. Rev. Lett. 84, 6082 (2000).

${ }^{20}$ S. J. Tans and C. Dekker, Nature (London) 404, 834 (2000).

${ }^{21}$ Single Charge Tunneling, edited by M. H. Grabert and M. H. Devoret (Plenum, New York, 1992). 